University of Wollongong

Research Online

Faculty of Engineering - Papers (Archive)

Faculty of Engineering and Information

Sciences

March 2005

\title{
Reversible Thermoelectric Nanomaterials
}

T. E. Humphrey

University of Wollongong

H. Linke

University of Oregon, USA

Follow this and additional works at: https://ro.uow.edu.au/engpapers

Part of the Engineering Commons

https://ro.uow.edu.au/engpapers/278

\section{Recommended Citation}

Humphrey, T. E. and Linke, H.: Reversible Thermoelectric Nanomaterials 2005.

https://ro.uow.edu.au/engpapers/278

Research Online is the open access institutional repository for the University of Wollongong. For further information contact the UOW Library: research-pubs@uow.edu.au 


\title{
Reversible Thermoelectric Nanomaterials
}

\author{
T. E. Humphrey ${ }^{1,2, *}$ and H. Linke ${ }^{3}$ \\ ${ }^{1}$ Engineering Physics, University of Wollongong, Wollongong 2522, Australia \\ ${ }^{2}$ School of Physics, University of New South Wales, New South Wales 2052, Australia \\ ${ }^{3}$ Materials Science Institute and Physics Department, University of Oregon, Eugene, Oregon 97403-1274, USA \\ (Received 20 July 2004; published 9 March 2005)
}

\begin{abstract}
Irreversible effects in thermoelectric materials limit their efficiency and economy for applications in power generation and refrigeration. While electron transport is unavoidably irreversible in bulk materials, here we derive conditions under which reversible diffusive electron transport can be achieved in nanostructured thermoelectric materials. We provide a fundamental thermodynamic explanation for why the optimum density of states in a thermoelectric material is a delta function and for why inhomogeneous doping and segmentation improve the thermoelectric figure of merit.
\end{abstract}

DOI: 10.1103/PhysRevLett.94.096601

The textbook examples for heat engines are cyclic engines, such as the well-known Carnot, Otto, and Brayton cycles, which may be used to model steam turbines or gasoline engines [1]. Cyclic heat engines transfer heat between at least two reservoirs via a working gas that moves through a number of quasiequilibrium states. Reversibility may be achieved when the cycle progresses infinitely slowly and heat transfer is isothermal [1].

Less well known is a distinct type, which we here denote as particle exchange (PE) heat engines [2]. These transfer heat between at least two reservoirs via the continuous exchange of particles in a finite energy range in the presence of a field against which work is done. Reversibility is achieved when particles are transmitted only at the energy where the occupation of states in the reservoirs is equal [35]; heat transfer is then isentropic but nonisothermal [2]. Most PE heat engines studied previously have been discrete, with particles moving elastically between two reservoirs only, and include the three-level amplifier [2,3], solar cells and light emitting diodes [2,4], and ballistic electron heat engines [2,5,6].

Nanostructured thermoelectric materials can have dramatically higher efficiencies than their bulk counterparts [7-11]. While the precise physical effects responsible for this improvement in certain materials are not well understood, theoretically it has been shown that the higher interface density in thermoelectric nanomaterials can reduce losses due to phonon heat conductivity [12] and also that a sharply peaked electronic density of states (DOS) (as may, in principle, be achieved in low-dimensional materials $[11,13,14])$ is optimal for high efficiency [15].

Here we model thermoelectric nanomaterials from a thermodynamic point of view as continuous PE heat engines in which electrons in a relatively narrow energy band move diffusively through a material with a continuous spatial variation in temperature and electrochemical potential. We derive conditions under which thermoelectric nanomaterials can operate reversibly, so challenging a long held view that thermoelectric devices are inherently
PACS numbers: 72.20.Pa, 05.70.-a, 73.63.Kv, 85.35.Be

irreversible heat engines [16]. Our results have significant potential for practical application in the design and optimization of new thermoelectric nanomaterials with very high limiting efficiencies.

The efficiency of any heat engine is bounded above by the Carnot limit, which can be achieved only in systems infinitesimally close to an equilibrium state. An electronic system in equilibrium is characterized by a spatially invariant occupation of states given by the Fermi-Dirac (FD) distribution function,

$$
f_{\mathrm{FD}}=\left[\exp \left(s^{+1}(\mathbf{r}) / k\right)+1\right]^{-1},
$$

where $s^{+1}(E, \mathbf{r})=[E-\mu(\mathbf{r})] / T(\mathbf{r})$ corresponds to the entropy change in the system if one electron with energy $E$ is added to the system at the spatial coordinate $\mathbf{r}$. A spatially invariant probability of occupation of available electronic states can be achieved in three ways, two of which are well known, while the third, a continuous form of the result for discrete PE heat engines, is pointed out here.

First, a state of global equilibrium is attained when the electrochemical potential $\mu(\mathbf{r})$ and the temperature $T(\mathbf{r})$ are both constant as a function of $\mathbf{r}$. This corresponds to the textbook definition of an equilibrated electronic system.

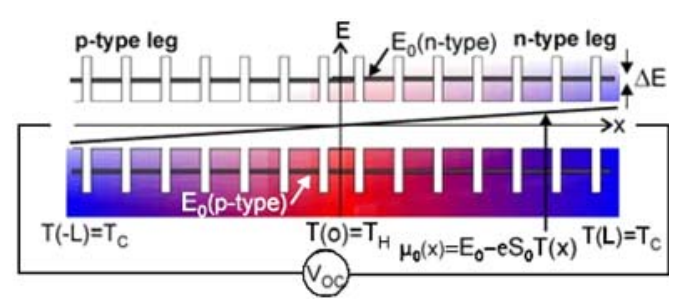

FIG. 1 (color online). Band structure of a thermoelectric quantum dot superlattice (QDSL) or a superlattice nanowire (SLNW) as used in our numerical model. Narrow minibands with width $\Delta E$ for electrons (holes) are located at energy $E_{0}\left(-E_{0}\right)$. The doping level varies across the material to ensure that $\mu(x)$ satisfies Eq. (3). Note that for simplicity, band bending near the metallic contacts has not been considered. 
Second, equilibrium can be approached when the only available electronic states in the system are at very high energies (for instance, in an intrinsic semiconductor with band gap, $E_{g} \rightarrow \infty$ ) where occupation tends to a spatially constant value of zero, irrespective of finite gradients in $\mu(\mathbf{r})$ and $T(\mathbf{r})$.

Here, we identify a third way in which equilibrium can be approached in continuous electronic systems, which we denote "energy-specific equilibrium" [17]. We consider a material in which (i) the DOS for electrons is a delta function at $E_{0}$ and in which (ii) $\mu(\mathbf{r})$ and $T(\mathbf{r})$ vary across the system in such a way that $s^{+1}\left(E_{0}, \mathbf{r}\right)$ is spatially invariant. This means that the population of electron states at the specific energy $E_{0}$ is the same throughout the material [see Figs. 1 and 2(c)]. Under these conditions the entire electronic system is in equilibrium, in spite of the thermal and potential gradients.

In the following we show how energy-specific equilibrium can be implemented in order to achieve reversible electron transport. We note that the rate of entropy production per unit volume, $\dot{s}$, in the material due to the movement of electrons in response to temperature and electrochemical potential gradients is [1]

$$
\dot{s}=\nabla\left(\frac{1}{T}\right)\left(J_{q}+\mu J_{n}\right)-\nabla\left(\frac{\mu}{T}\right) J_{n}
$$

where $J_{n}$ is the number flux of electrons in the direction of decreasing temperature and $J_{q}$ the heat flux due to electrons, and where $\mu, T$, and $J_{q}$ depend on $\mathbf{r}$. In the limit that only electrons with energy $E_{0}$ are transmitted, the heat current in the material is given by $J_{q}(\mathbf{r})=J_{n}\left[E_{0}-\right.$ $\mu(\mathbf{r})]$. To find the conditions under which electrons move

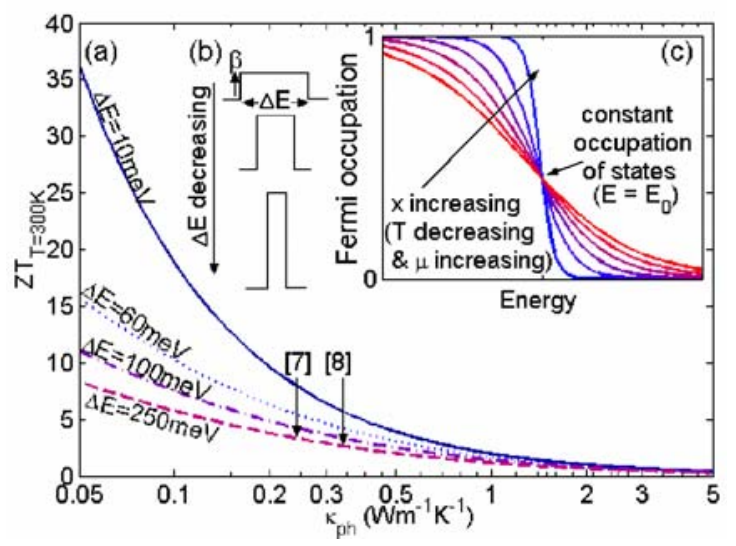

FIG. 2 (color online). (a) $Z T$ at $T_{C}=300 \mathrm{~K}$ as a function of $\kappa_{\mathrm{ph}}$ for different $\Delta E$ as indicated. Experimental values are marked [7] and [8] for $\kappa_{\mathrm{ph}}$ obtained at $300 \mathrm{~K}$ in the nanomaterials described in the corresponding references. (b) Schematic of the variation of the magnitude of $\beta$ with decreasing $\Delta E$ resulting from the scaling operation described in the caption to Fig. 3. (c) Fermi occupation function at several places along the $x$ axis in the material shown in Fig. 1. through the material without increasing the entropy of the system (reversible transport) we set $\dot{s}\left(E_{0}\right)=0$ to obtain a differential equation which may be solved by integration with respect to $\mathbf{r}$ to find $E_{0}=\mu(\mathbf{r})+\Omega T(\mathbf{r})$. Here $\Omega$ is an integration constant, evaluated using the boundary condition $\mu_{C}-\Omega T_{C}=\mu_{H}-\Omega T_{H}$, where the subscripts $C$ and $H$ refer to the cold and hot extremes of the system, respectively. At open circuit, this yields $\Omega=-e V_{\mathrm{OC}} / \Delta T$, where $e V_{\mathrm{OC}}=\mu_{C}-\mu_{H}, \Delta T=\left(T_{H}-T_{C}\right)$, and where $\Delta T$ and $\mu_{C}$ (or alternatively $\mu_{H}$ ) are freely chosen parameters. We then arrive at our main result, namely, an expression for a spatially varying chemical potential that ensures that electron transport at $E_{0}$ is reversible at open circuit:

$$
\mu_{0}(\mathbf{r})=E_{0}-e V_{\mathrm{OC}}[T(\mathbf{r}) / \Delta T] .
$$

(The subscript 0 refers to the state of energy-specific equilibrium.) To clarify the physics involved in Eq. (3) we note that

$$
S_{0} \equiv V_{\mathrm{OC}} / \Delta T=\left[E_{0}-\mu_{0}(\mathbf{r})\right] / e T(\mathbf{r})
$$

is the thermopower (Seebeck coefficient) corresponding to energy-specific equilibrium, which can be physically interpreted as the entropy carried by $1 \mathrm{~A}$ of current [1] [that is, $\left.e S_{0}=s^{+1}\left(E_{0}\right)\right]$. As $S_{0}$ is spatially invariant, there is no entropy increase when an electron with energy $E_{0}$ moves through the material and, therefore, no thermodynamically spontaneous direction for current to flow, despite the finite thermal and electrical potential gradients, confirming that the electronic system is in equilibrium.

In practice, reversible electron transport can be approached by (i) creating a nanostructured material, such as a QDSL or a SLNW $[9,18]$ with a DOS for mobile electrons that is sharply peaked at one energy $E_{0}$ [which could, in principle, depend on $\mathbf{r}$ so that $E_{0}=E_{0}(\mathbf{r})$ [19] ] and that is (ii) inhomogeneously doped such that Eq. (3) is fulfilled (Fig. 1) [19]. Note that a given material can be optimized only for one specific temperature gradient.

A material with a delta function DOS doped according to Eq. (3) will have an "electronic" efficiency (efficiency in the absence of phonon heat leaks and other nonidealities) approaching the Carnot limit. To show this, we note that the heat flux withdrawn from the hot end of the system by electrons is $\left|J_{q}\left(T_{H}\right)\right|=\left(E_{0}-\mu_{H}\right)\left|J_{n}\right|$ [Eq. (3)]. At open circuit the power is $P \equiv e V_{\mathrm{OC}}\left|J_{n}\right|=e S_{0}\left(T_{H}-T_{C}\right)\left|J_{n}\right|$, giving an electronic efficiency $\eta_{\mathrm{PG}} \equiv P / J_{q}\left(T_{H}\right)=$ $\left(1-T_{C} / T_{H}\right)$, which is the Carnot limit for power generation. Similarly, if the system is operated in reverse $\left(J_{n} \rightarrow\right.$ $\left.-J_{n}\right)$ as a refrigerator, then the coefficient of performance can be shown to be equal to the Carnot limit, $\eta_{R} \equiv$ $J_{q}\left(T_{C}\right) / P=\left[T_{C} /\left(T_{H}-T_{C}\right)\right]$.

$S_{0}$ is, in fact, the theoretical upper bound upon the Seebeck coefficient $S(\mathbf{r})$ for a particular value of $\left[E_{0}-\right.$ $\mu(\mathbf{r})]$ and $T(\mathbf{r})$. To show this, we set $\mu(\mathbf{r})=\mu_{H}$ and $T(\mathbf{r})=$ $T_{H}$ and assume that a distance $\delta \mathbf{r}$ from $\mathbf{r}$ the potential in- 
crease is $\delta \mu$ and the temperature decrease is $\delta T$. Then using Eq. (4) we find that $e S_{0} \delta T=\left(E_{0}-\mu_{H}\right) \delta T / T_{H}$. This means that $S=S_{0}$ when the Carnot fraction, $\delta T / T_{H}$, of heat removed by each electron with energy $E$ from the hot end of the small section of material, $\left(E_{0}-\right.$ $\left.\mu_{H}\right)$, is converted to useful work $e S_{0} \delta T=\delta \mu . S_{0}$ therefore represents a theoretical maximum, as $S>S_{0}$ would imply an efficiency greater than the Carnot limit.

It is important to note that the presence of inelastic scattering in thermoelectric materials does not affect these results. Inelastic scattering processes produce heating via the relaxation of carriers from a nonequilibrium occupation of states to an occupation given by a FD distribution with the appropriate local value of $\mu(\mathbf{r})$ and $T(\mathbf{r})$. Since in our system states at $E_{0}$ are occupied with the same probability throughout the material [see Fig. 2(c)], inelastic processes that scatter individual electrons into these states are just as likely as processes that scatter electrons out of these states, so there is no net exchange of energy between free electrons and the lattice due to the movement of electrons with energy $E_{0}$.

If the DOS is finite in some range around $E_{0}$, electrons occupying states at energies above (below) $E_{0}$ are, on average, scattered to lower (higher) energy states, depending upon the difference between the local probability of occupation and that of the arriving electrons. This process results in local heating (cooling) of the lattice. Crucially, however, the nearer these electrons are in energy to $E_{0}$, the smaller the heating (cooling) effect, due to the small variation in the occupation of states between adjacent regions of the material.

To quantify the advantage of using a deltalike DOS and inhomogeneous doping according to Eq. (3), we now numerically characterize a nanomaterial in which we vary the DOS from deltalike to bulklike. We assume a finite lattice thermal conductivity $\left(\kappa_{\mathrm{ph}} \neq 0\right)$ and a single miniband of width $\Delta E$ as shown in Fig. 1. We use the Boltzmann transport equation under the relaxation-time approximation [20]. Electrical conductivity, $\sigma$, thermal conductivity due to electrons, $\kappa_{\mathrm{el}}$, and $S$ can all be expressed as a function of the integral [20]

$$
K_{\alpha}=\int \beta(E)(E-\mu)^{\alpha}\left(-\frac{d f}{d E}\right) d E
$$

where $\beta(E)=D(E) \tau(E) \nu(E)^{2}, \tau(E)$ is the electron relaxation time, $\nu(E)$ is the electron group velocity, $D(E)$ is the DOS, and where $\alpha=0,1$, or 2. Then $\sigma=e^{2} K_{0}, S=$ $-K_{1} /\left(e T K_{0}\right), \kappa_{\mathrm{el}}=\left(K_{2}-K_{1}^{2} / K_{0}\right) / T$, and the dimensionless figure of merit $Z T=T \sigma S^{2} /\left(\kappa_{\mathrm{el}}+\kappa_{\mathrm{ph}}\right)$. We use $\kappa_{\mathrm{ph}}=$ $0.33 \mathrm{~W} \mathrm{~m}^{-1} \mathrm{~K}^{-1}$, the value measured by Harman et al. for $\mathrm{PbSeTe} / \mathrm{PbTe}$ QDSL [8]. For simplicity and transparency in the numerical results, we assume that $\beta(E)=\beta$ is constant over the energy range $\Delta E$, a reasonable assumption for the small values of $\Delta E$ in which we are primarily interested. To isolate the effect upon $Z T$ of changing $\Delta E$ from effects due to changing the overall number of states, we scale the magnitude of $\beta$ with $\Delta E$ such that for all values of $\Delta E, \sigma=5 \times 10^{5} \Omega^{-1} \mathrm{~m}^{-1}$ at $\mu_{c}=E_{0}$ [see Fig. 2(b) and the Fig. 3 caption for details]. This choice means that at the values of $\mu_{C}$ for which $Z T$ is optimized at $T_{C}=300 \mathrm{~K}$, we obtain $\sigma \approx 6 \times 10^{4} \Omega^{-1} \mathrm{~m}^{-1}$ for $\Delta E \approx$ $200 \mathrm{meV}$, the same conductivity as measured in [8] (note that the energy spectrum of the DOS in [8] is not known). In addition, we obtain $\sigma \approx 1 \times 10^{5} \Omega^{-1} \mathrm{~m}^{-1}$ for an optimized $Z T$ at $\Delta E \approx 60 \mathrm{meV}$, which is similar to the value obtained by numerical modeling in [9] for a $\mathrm{PbSe} / \mathrm{PbS}$ SLNW with a $60 \mathrm{meV}$ wide miniband.

Figure 3(a) shows $S$ as a function of $\left(E_{0}-\mu_{C}\right)$ at the position $x=L$ for different $\Delta E$. As $\Delta E$ decreases the system approaches energy-specific equilibrium and $S$ approaches $S_{0}$, the theoretical maximum Seebeck coefficient given by Eq. (4).

Figures 3(b) and 3(c) show $\sigma$ and $\kappa_{\mathrm{el}}$, respectively, as a function of $\left(E_{0}-\mu_{C}\right)$. Note that although $\sigma$ at $\mu_{C}=E_{0}$ is kept constant with decreasing $\Delta E, \kappa_{\mathrm{el}}$ at $\mu_{C}=E_{0}$ decreases; that is, $K_{1}{ }^{2} / K_{0} \rightarrow K_{2}$ [15]. The physical reason for this is that as the heat carried by an electron is proportional to the difference between its energy and the Fermi energy, materials with narrow DOS $(\Delta E / 2$ less than several $k T$ ), which "cut off" the high energy end of the Fermi distribution, have low $\kappa_{\mathrm{el}}$. An important implication of this result is that the Wiedemann-Franz law, which expresses that $\kappa_{\mathrm{el}} / \sigma T=(\kappa \pi)^{2} / 3 e^{2}$ [20], and which is often used by experimentalists to calculate $\kappa_{\mathrm{el}}$ from the electrical conductivity $[7,8]$, loses validity in nanomaterials with narrow DOS. Crucially, this result also means that thermoelectric nanomaterials are not subject to the conundrum that limits
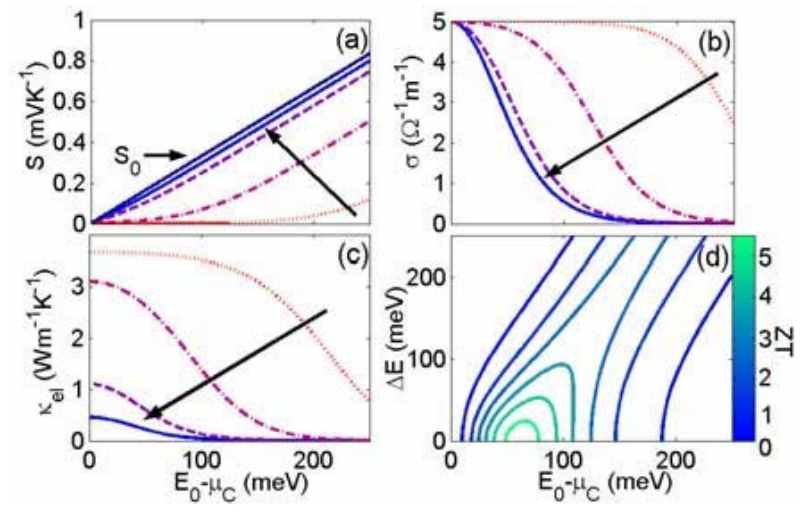

FIG. 3 (color online). Numerically calculated thermoelectric parameters as a function of $\left(E_{0}-\mu_{C}\right)$ for $\Delta E=500,250,100$, and $60 \mathrm{meV}$ (arrows indicate decreasing $\Delta E$ ), for $T(L)=$ $300 \mathrm{~K}$, and for $\kappa_{\mathrm{ph}}=0.33 \mathrm{Wm}^{-1} \mathrm{~K}^{-1}$. For each $\Delta E$ we used the value $\beta=5 \times 10^{5} / e^{2} K_{0}\left(\Delta E, \mu_{c}=E_{0}\right)$ to calculate $K_{0}, K_{1}$, and $K_{2}$ for $\mu_{c} \neq E_{0}$. See the text for further model details. (a) Seebeck coefficient. (b) Electrical conductivity. (c) Electronic thermal conductivity. (d) Contour map of ZT. (a) is antisymmetric and (b) $-\left(\right.$ d) mirror symmetric with respect to $\left(E_{0}-\right.$ $\left.\mu_{C}\right)=0$ (not shown). 
$Z T$ of bulk thermoelectrics: the interrelationship of $S, \sigma$, and $\kappa_{\mathrm{el}}$.

To emphasize this point, we show in Fig. 3(d) that for small $\Delta E$ the figure of merit is optimal at values of $\left(E_{0}-\mu_{C}\right)$ [21] where $S$ [Fig. 3(a)] and $\kappa_{\mathrm{el}}$ [Fig. 3(c)] are both relatively small. In other words, the best strategy to maximize $Z T$ in nanomaterials is to minimize $\kappa_{\mathrm{el}}$ leaving $Z T$ limited only by finite $\kappa_{\mathrm{ph}}$. The fact that this is possible without at the same time decreasing $\sigma$ illustrates a fundamental difference between the thermodynamics of nanostructured and bulk thermoelectric materials.

Using the technique outlined in [22], the difference between keeping $\left[E_{0}-\mu(x)\right] / T(x)$ constant across a thermoelectric nanomaterial [i.e., $\mu(x)=\mu_{0}(x)$ ] via inhomogeneous doping or segmentation and allowing $\left[E_{0}-\mu(x)\right] / T(x)$ to vary [i.e., $\left.\mu(x)=\mu_{0}(L / 2)\right]$ can be quantified. We find [23] that keeping $\left[E_{0}-\mu(x)\right] / T(x)$ constant can increase the maximum efficiency by $10 \%$ (corresponding to a doubling of $Z T$ at $300 \mathrm{~K}$ for $\Delta E=10 \mathrm{meV}, T_{H}=800 \mathrm{~K}, T_{C}=300 \mathrm{~K}$, and $\kappa_{\mathrm{ph}}=$ $0.5 \mathrm{~W} \mathrm{~m}^{-1} \mathrm{~K}^{-1}$ ) and increase the maximum power by up to $60 \%$. The physics behind this improvement is now clear; inhomogeneous doping and segmentation increase $Z T$ as they bring thermoelectric materials closer to a state of energy-specific equilibrium.

Figure 2(a) shows $Z T$ at $300 \mathrm{~K}$ as a function of $\kappa_{\mathrm{ph}}$, demonstrating that while a decrease in $\kappa_{\mathrm{ph}}$ is always beneficial to the figure of merit, a decrease in $\kappa_{\mathrm{ph}}$ combined with a decrease in $\Delta E$ results in spectacular increases in $Z T$. The development of nanomaterials with a $\kappa_{\mathrm{ph}}$ that is $20 \%$ lower than in current state-of-the-art materials and optimized, deltalike DOS could result in $Z T=10$ at $T=$ $300 \mathrm{~K}$, well within the range of $Z T>5$ required for economical adoption of thermoelectric technology for mainstream refrigeration and power generation.

Finally, we note the finite coherence length of electrons, which places a lower limit on the width of DOS peaks resulting from quantum confinement, will also, in principle, limit the efficiency of thermoelectric nanomaterials. However, as all heat engines are in reality operated at finite power, away from maximum efficiency, quantum efficiency limits are not expected to be a practical design issue.

We thank H. J. Goldsmid for helpful comments on the manuscript. T.H. is supported by the ARC and SERDF, and H. L. by NSF (PHY 0239764).

*Electronic address: tammy.humphrey@unsw.edu.au

[1] Herbert B. Callen, Thermodynamics and an Introduction to Thermostatics (Wiley, New York, 1985).
[2] T.E. Humphrey and H. Linke, cond-mat/0407508 [Physica (Amsterdam) E (to be published)].

[3] H.E. D. Scovil and E. O. Schulz-DuBois, Phys. Rev. Lett. 2, 262 (1959).

[4] P. T. Landsburg and G. Tonge, J. Appl. Phys. 51, R1 (1980).

[5] T.E. Humphrey, R. Newbury, R. P. Taylor, and H. Linke, Phys. Rev. Lett. 89, 116801 (2002).

[6] T.E. Humphrey and H. Linke, cond-mat/0401377.

[7] R. Venkatasubramanian, E. Siivola, T. Colpitts, and B. O'Quinn, Nature (London) 413, 597 (2001).

[8] T. C. Harman, P. J. Taylor, M. P. Walsh, and B. E. LaForge, Science 297, 2229 (2002).

[9] Yu-Ming Lin and M. S. Dresselhaus, Phys. Rev. B 68, 075304 (2003).

[10] K. F. Hsu, S. Loo, F. Guo, W. Chen, J. S. Dyck, C. Uher, T. Hogan, E. K. Polychroniadis, and M. G. Kanatzidis, Science 303, 818 (2004).

[11] D. Bilc, S. D. Mahanti, K. F. Hsu, E. Quarez, R. Pcionek and M. G. Kanatzidis, Phys. Rev. Lett. 93, 146403 (2004).

[12] D. G. Cahill, W. K. Ford, K.E. Goodson, G. D. Mahan, A. Majumdar, H. J. Maris, R. Merlin, and S. R. Phillpot, J. Appl. Phys. 93, 793 (2003).

[13] L.D. Hicks and M.S. Dresselhaus, Phys. Rev. B 47, 12727 (1993)

[14] L.D. Hicks and M.S. Dresselhaus, Phys. Rev. B 47, 16631 (1993).

[15] G. D. Mahan and J. O. Sofo, Proc. Natl. Acad. Sci. U.S.A. 93, 7436 (1996).

[16] H. Littman and B. Davidson, J. Appl. Phys. 32, 217 (1961).

[17] Our conclusions are equally valid for continuous or discrete systems in which particles obey Bose-Einstein or Maxwell-Boltzmann statistics.

[18] High-yield bulk fabrication of SNLW should be possible using heterostructured whiskers as described in C. Thelander, T. Mårtensson, M. T. Björk, B. J. Ohlsson, M.W. Larsson, L.R. Wallenburg, and L. Samuelson, Appl. Phys. Lett. 83, 2052 (2003).

[19] Note that it is $\left[E_{0}-\mu\right]$ which appears in all the equations, so it would also be possible to satisfy Eq. (3) by varying $E_{0}$ across the material, for example, by changing the size of the quantum dots or by varying the band gap of the larger band gap material (for over-the-barrier transport).

[20] N.W. Ashcroft and N.D. Mermin, Solid State Physics (Saunders College Publishing, Orlando, FL, 1976).

[21] In Ref. [15] it is shown that the figure of merit of a thermoelectric material with a $\delta$-function DOS is optimized at $\left(E_{0}-\mu_{C}\right)=2.4 \mathrm{kT}(\approx 62 \mathrm{meV}$ at $300 \mathrm{~K})$.

[22] G. D. Mahan, J. Appl. Phys. 70, 4551 (1991).

[23] T.E. Humphrey and H. Linke, cond-mat/0407506 [Proceedings of the International Thermoelectric Conference, Adelaide, 2004 (to be published)]. 